\title{
Optimizing the magnetic circuit of an actuator
}

\author{
Lizeta Popescu ${ }^{1}$, and Lucian Diodiu ${ }^{2, *}$ \\ 1 "Lucian Blaga" University of Sibiu, Engineering Faculty, Computer and Electric Engineering \\ Department, Emil Cioran Street, No. 4, 550025 Sibiu, Romania \\ ${ }^{2}$ Energoconsult, Viile Sibiului no. 1, Sibiu, Romania
}

\begin{abstract}
The paper presents a modern method of constructive optimization of a plunger actuator with axial symmetry based on the electromagnetic simulation of several variants of magnetic circuit and excitation currents. On the basis of the force calculation developed by this actuator and the magnetic induction values in the core, we calculated the kinematic actuator parameters. Experimental determinations confirmed the precision and utility of the optimization method by electromagnetic simulation of the actuator.
\end{abstract}

\section{Actuator}

A modern method of assisted design of electrical equipment and most electromechanical converters consists of their analytical design and their optimization by electromagnetic and thermal simulations. The goal is to reduce the time and cost of designing and building prototypes.

Electric actuators are used for automation in a multitude of applications. In the automotive industry, it is used in the gearbox with a flange of the electric motor with integrated control, the control gearshift and the communication interface with the distributed control system. The benefits of the digital actuator solution include the reduction of cables (two-wire solution) and extended functionality.

In the current automotive industry, actuators (servomotors) are components not found in motorcycle engines, being responsible for strangulation valves (for regulating the air and fuel mixture by using a butterfly valve) or reel speed control.

The cinematic performance of a vehicle depends to a large extent on the performance of the actuator, and the scientific research in the field seeks to develop great powers, miniaturization, robustness, high efficiency and easy control of them.

Modern actuators are equipped with microcontrollers that monitor the internal state, also allowing for independent monitoring of various operations and conditions, including predefined operation times or the number of start-ups. [1-2]

The actuator is a temporary magnet (electromagnet of c.c.), whose action, attracting or releasing a ferromagnetic armature is determined by the presence of electric current in an excitation circuit. The actuator converts electrical energy into mechanical energy through magnetic energy, having three structure components: electrical, magnetic and mechanical.

Its optimal design is relatively difficult with a low degree of observability and controllability, because in dynamic mode we have the overlapping of an electric transient

\footnotetext{
${ }^{*}$ Corresponding author: lucian_diodiu@yahoo.com
} 
regime with a kinematic one. In this dynamic regime the reluctance of the magnetic circuit depends on the variable air gap, the time variation of the current and the magnetization curve. In these conditions, only numerical simulations have a satisfactory precision [3].

The differential equation of the actuator dynamic mode, taking into account the variation of inductance in relation to time, is:

$$
\mathrm{U}=\mathrm{R} \cdot \mathrm{i}+\mathrm{L} \cdot \frac{\mathrm{di}}{\mathrm{dt}}+\mathrm{i} \cdot \frac{\mathrm{dL}}{\mathrm{dt}}
$$

For such magnetic circuits, the inductivity is defined by the equation:

$$
\mathrm{L}_{\mathrm{m}}=\frac{\mathrm{d} \Psi}{\mathrm{di}}=\frac{\partial \Psi}{\partial \mathrm{i}}+\frac{\partial \Psi}{\partial \mu} \cdot \frac{\mathrm{d} \mu}{\mathrm{di}}+\sum_{\mathrm{j}=1}^{\mathrm{m}} \frac{\partial \Psi}{\partial \mathrm{x}_{\mathrm{j}}} \cdot \frac{\mathrm{dx}_{\mathrm{j}}}{\mathrm{di}}
$$

Where:

$-\Psi=\Psi\left(\mathrm{I}, \mu, \mathrm{x}_{\mathrm{j}}\right)$, is the magnetic flux;

- I is the current through the excitation coil;

- $\mu$ is the magnetic permeability of the core;

$-\mathrm{x}_{\mathrm{j}}$, is the general coordinates which determine the geometry of the magnetic circuit.

The equation (2) defined the "motion-dependent inductivity" as a generalization of the dynamic inductivity:

Experimental variation of electromagnetic and cinematic quantities is shown in fig 1.

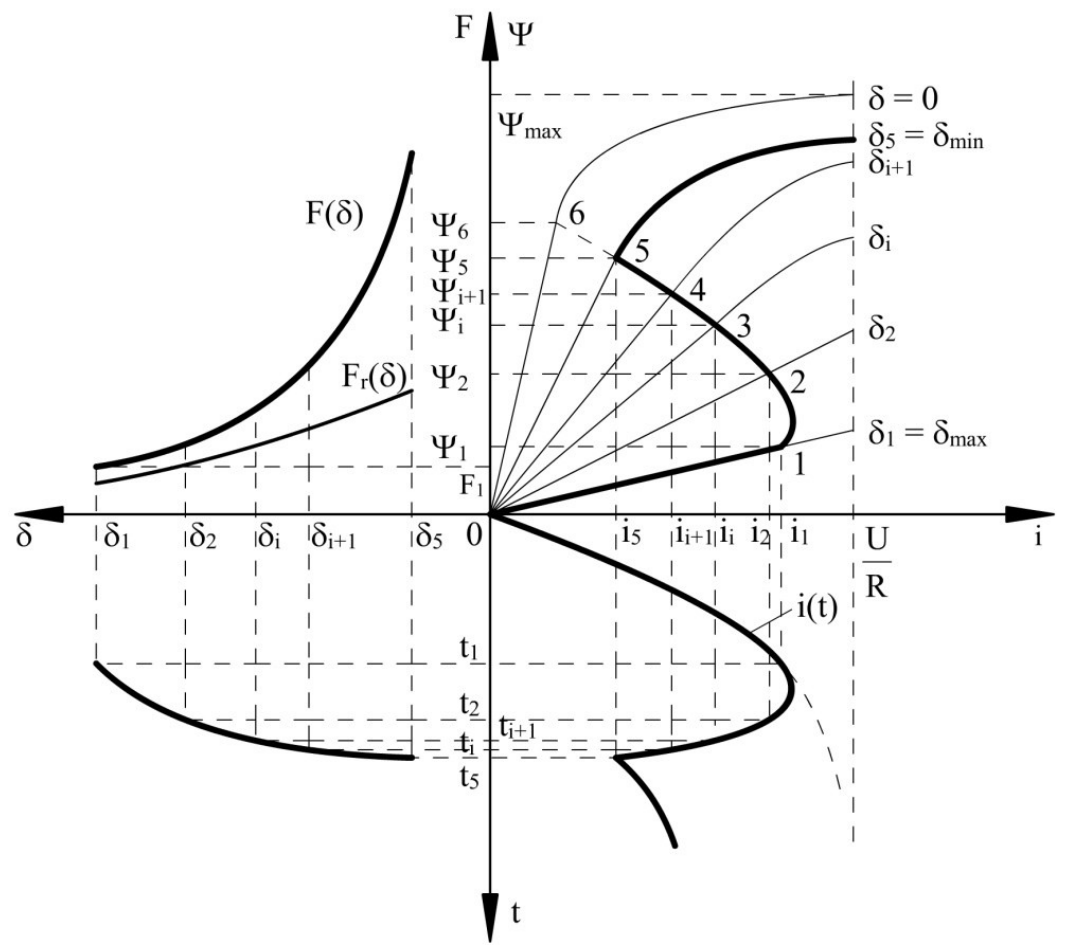

Fig. 1. Variation of electromagnetic and kinematic parameters in the actuator dynamic mode [1].

The calculation of the force developed by the actuator is done with the formula:

$$
\mathrm{F}(\mathrm{x}, \mathrm{i})=\oiint_{\Sigma}\left[\frac{1}{\mu_{0}} \overline{\mathrm{B}}(\overline{\mathrm{B}} \cdot \overline{\mathrm{n}})-\frac{1}{2 \cdot \mu_{0}} \mathrm{~B}^{2} \overline{\mathrm{n}}\right] \mathrm{d} \overline{\mathrm{s}}
$$


It results that numerical simulation of the dynamic regime is required for an acceptable precision of the force calculation and its design. [2]

\section{Electromagnetic simulation}

The electromagnetic simulation is designed to determine the variation of the magnetic induction in the actuator and to obtain the value of the electromagnetic force in its moving parts. By making the electromagnetic simulation, we can determine the state of the actuator from a magnetic point of view, so in case of inappropriate results (too small electromagnetic force, magnetic induction too close to saturation, etc.), we are oriented towards optimizing the results. [2]

In the electromagnetic simulation, we will only use magnetic stator parts of the stator, namely the iron parts (the stator and the movable part of the iron), the coils and the permanent magnets.

Of the three numerical modeling methods (finite difference method, finite element method and boundary element method) we have chosen the finite element method

The basic idea of the finite element method is to find the solution to a complicated problem by replacing it with a simpler one. We have opted for direct formulation based on the matrix calculation of structures using the displacement method. [5]

We opted for Comsol Multiphysics 4.3 which is a powerful interactive environment for modeling and solving all kinds of scientific and engineering problems. The program is based on the theory of the finite element, based on the 2 nd order differential equations.

By using Comsol physical interface and advanced material properties, it is possible to construct models based on the definition of relevant physical quantities, such as material properties, forces, constraints, sources and flows. We had access to Comsol Muliphysics through a flexible graphical interface (GUI), Java programming script or Matlab. [5]

In order to electromagnetically simulate the actuator we want to optimally constructively we need its 3D mechanical design. Computer-aided mechanical design can be done in software such as Catia, SolidWorks, Solidedge, Inventor, AutoCAD, etc. We chose CATIA V5ale IBM PLM (Product Lifecycle Management) solutions that meet the requirements and exigencies.

Steps to achieve electromagnetic simulation in Comsol Multiphysics:

1. The Comsol Multiphysics program opens, the opening will show the workspace,

2. Choose the dimensional space ( $2 \mathrm{D}$ axially symmetrical), then choose the physical model and type of the study to be simulated.

3. The geometry of the model to be simulated will be created or will be imported if it was previously created in the Catia V5 program.

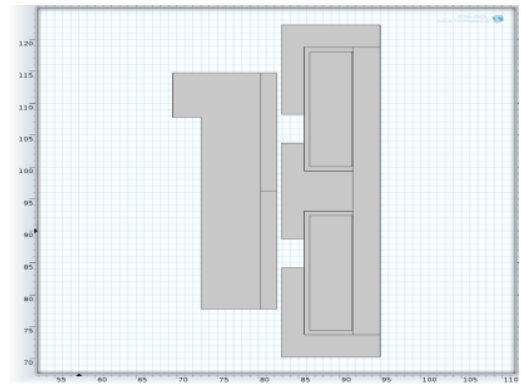

Fig. 2. Creating actuator geometry.

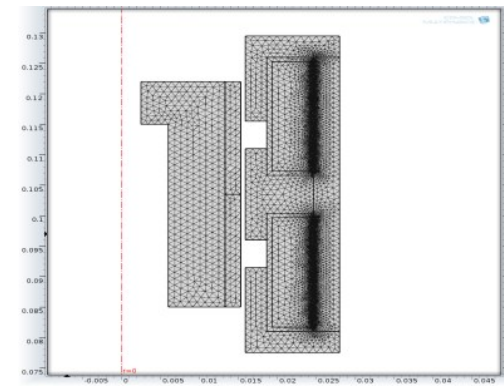

Fig. 3. Making the Mesh.

4. The global parameters and variables are added to the program under the Global Definitions or Variables sections.

5. Add the materials from which the track to be simulated is built, along with all material properties and definition laws. 
6. Add the physical laws that will intervene in the simulation (for example, in the case of an electrothermal simulation, the initial temperature, the heat source range, the heat flow, the thermal insulation, the thermal radiation with the ambient etc.)

7. Mesh density is chosen based on the precision required for optimal results. Finite elements are triangular.

8. Setting study characteristics: parameterization, simulation duration (in the case of transient simulations), external variables, etc.

9. Processing the results. [1]

The simulation was performed for 4, 8 and 12 A currents. An axial magnetization with a retention of $1.1 \mathrm{~T}$ was chosen for the same direction for both magnets and a winding in the same direction for both coils. Since electromagnetic simulations only concern magnetic components, we only use the stator, coils, magnets, and magnet corresponding to the magnets. The actuator was studied once by reducing the air gap by increasing the electromagnetic curves by about $15 \mathrm{~N}$ for each current and the second by increasing the coils by $0.25 \mathrm{~mm}$ in each direction. From the graphs resulted an increase of the electromagnetic force with about $30 \mathrm{~N}$ for each current, and the third time combined by both reducing the $2 \mathrm{~mm}$ air gap and increasing the busses by $0.25 \mathrm{~mm}$ each, resulting in higher electromagnetic forces of about $40 \mathrm{~N}$ per current. [4]

A first optimization would be to change the magnet magnetization direction from AXIAL to RADIAL and the opposite direction of one magnet to the other. This type of optimization will be done preliminarily for all three actuators, after which constructive optimizations will be made.
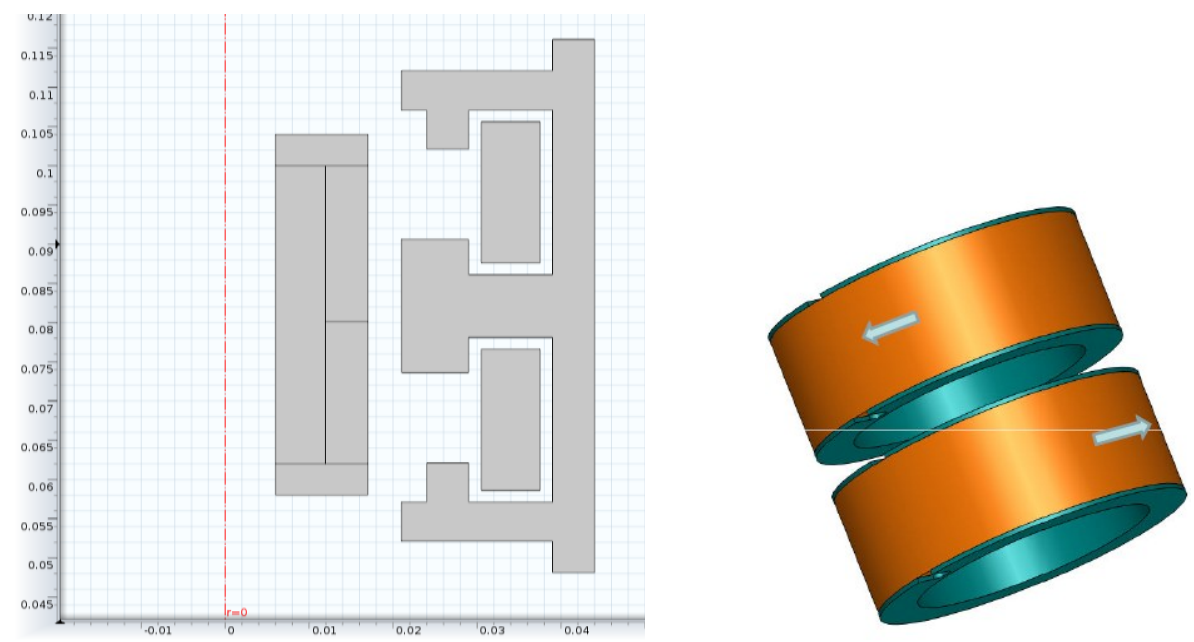

Fig. 4. Input data in magnetic simulations for actuator optimization. 


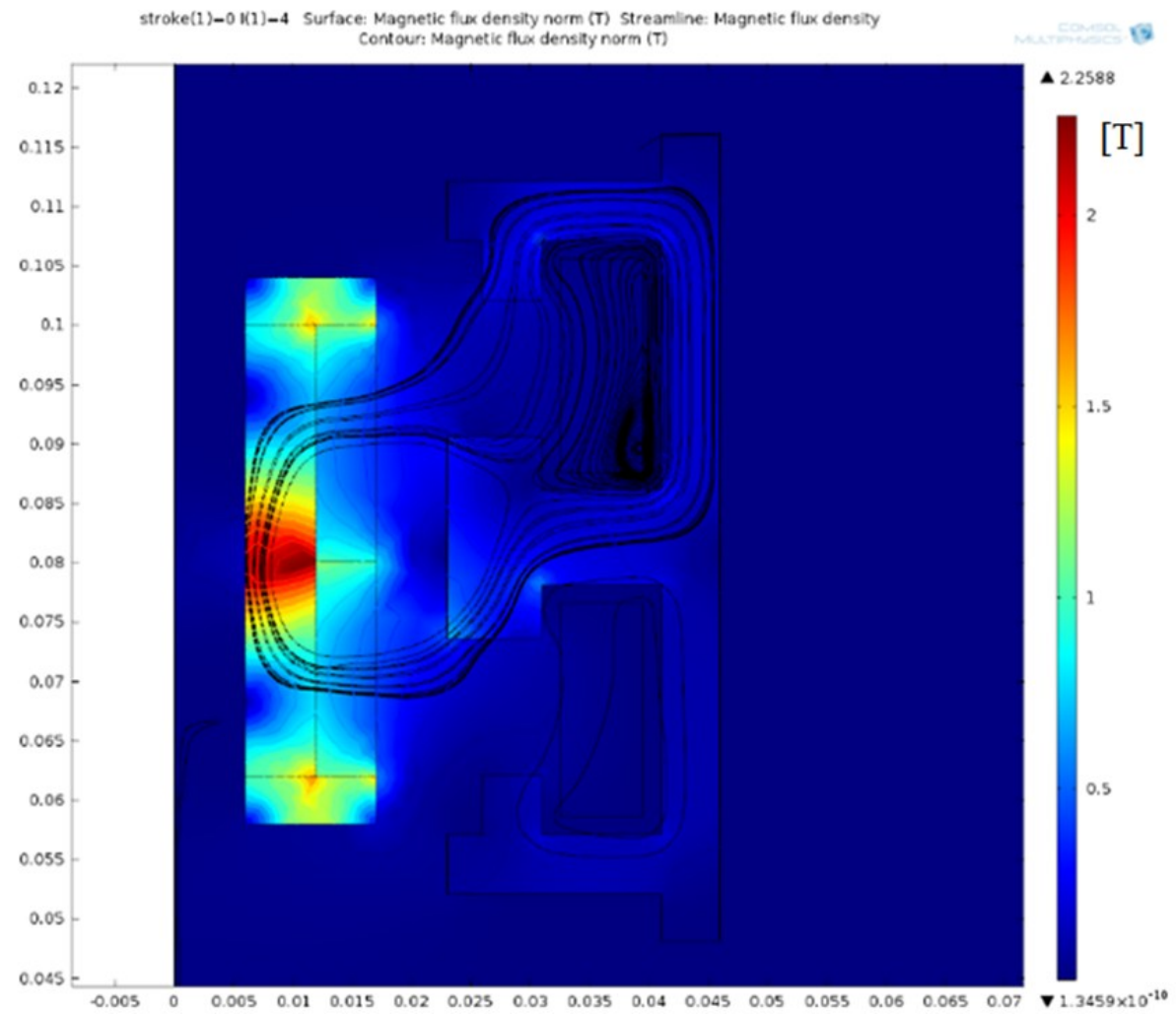

Fig. 5. Output of electromagnetic actuator simulation for $4 \mathrm{~A}$.
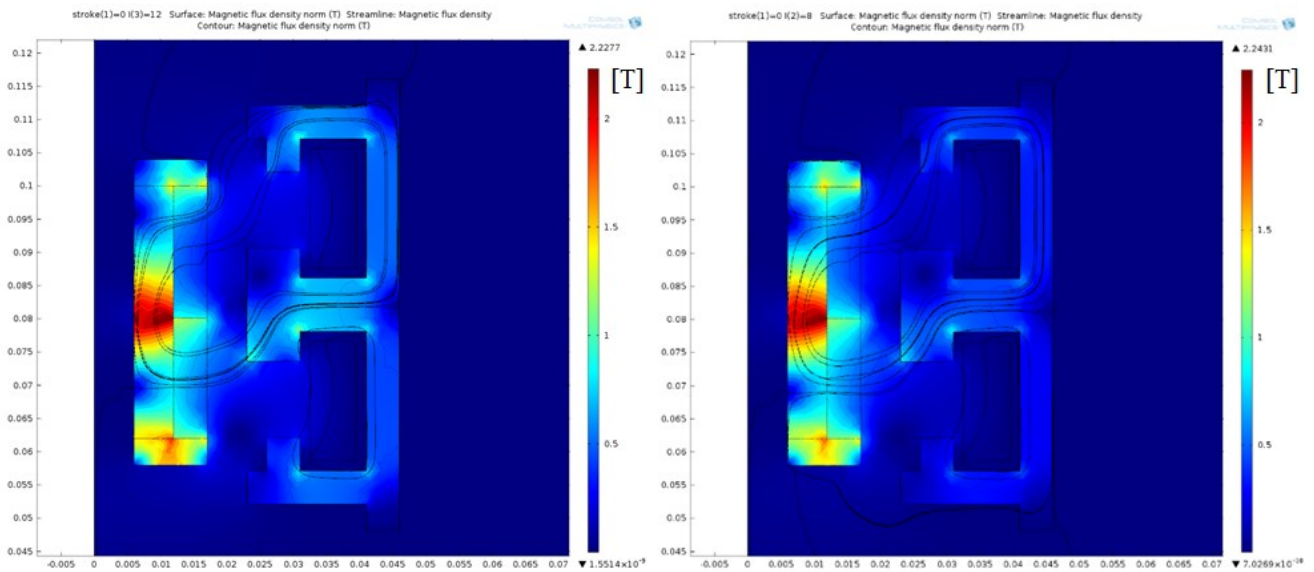

Fig. 6. Output of electromagnetic actuator simulation for 8 A and $12 \mathrm{~A}$.

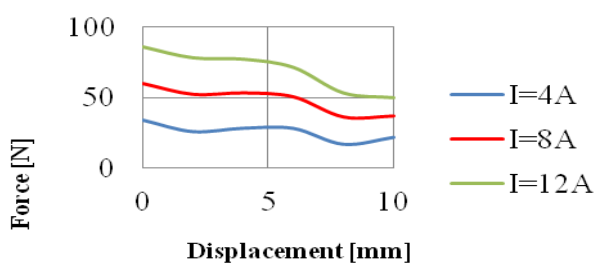

Fig. 7. Drive-to-Actuator Chart for 4, 8, $12 \mathrm{~A}$ 


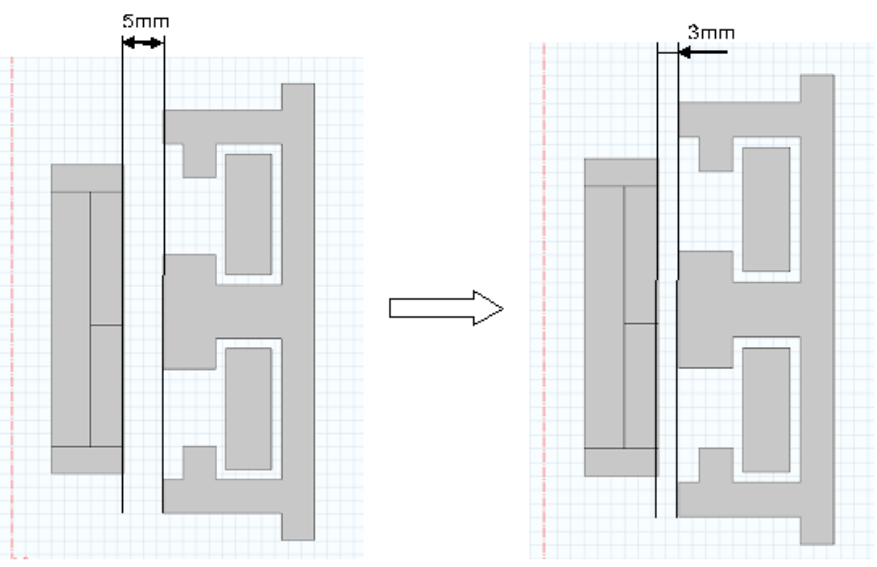

Fig. 8. Reduce the air gap by $2 \mathrm{~mm}$

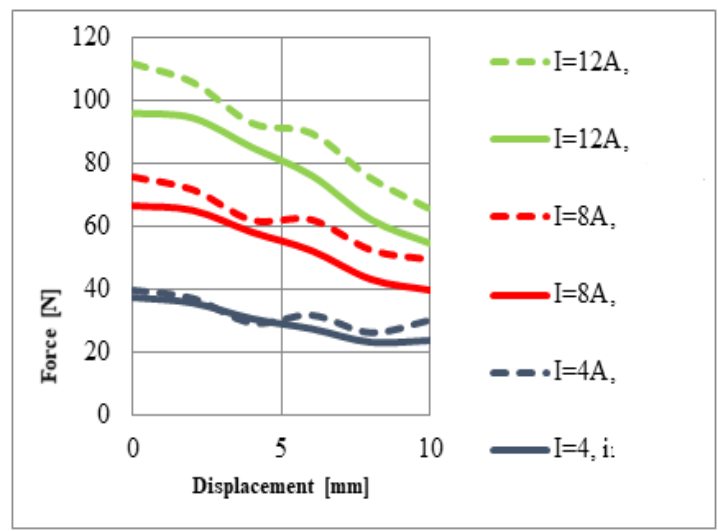

Fig. 9. Force-displacement chart. Comparison between force at initial air gap and reduced air gap by 2 mm Initial air gap Reduced air gap
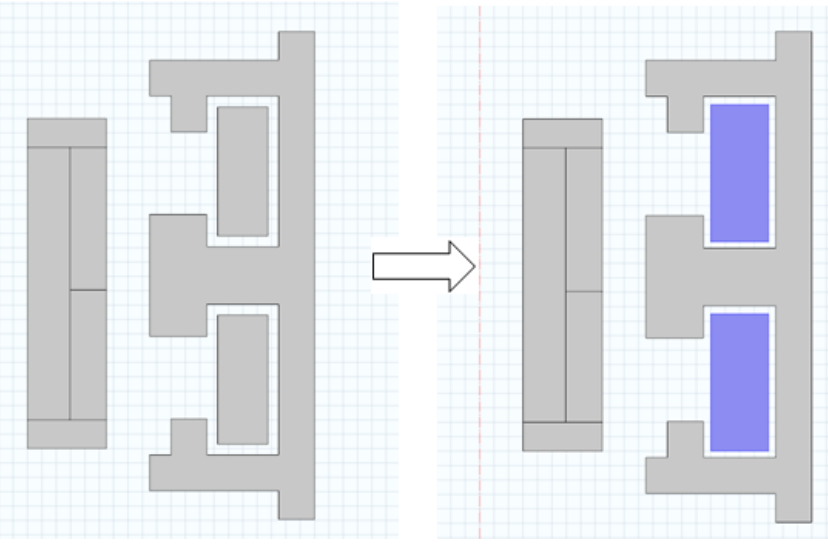

Fig. 10. Increase coils by $0.25 \mathrm{~mm}$ in each direction 


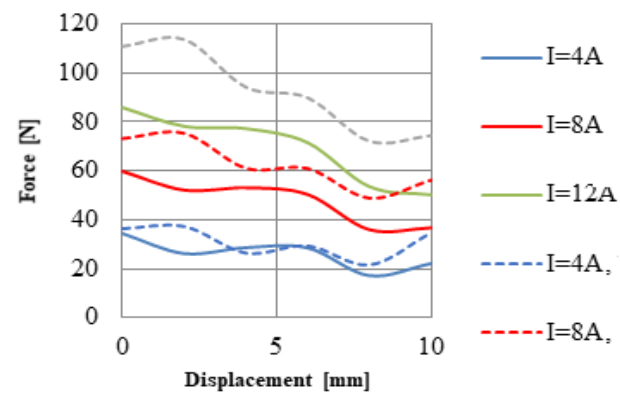

Fig. 11. Force-displacement chart after coil magnification Enlarged coils.

Figure 12 shows the optimized actuator model. It is of major interest in the automotive industry because the electromagnetic force supplied by it is a linear one and relatively large compared to its dimensions.

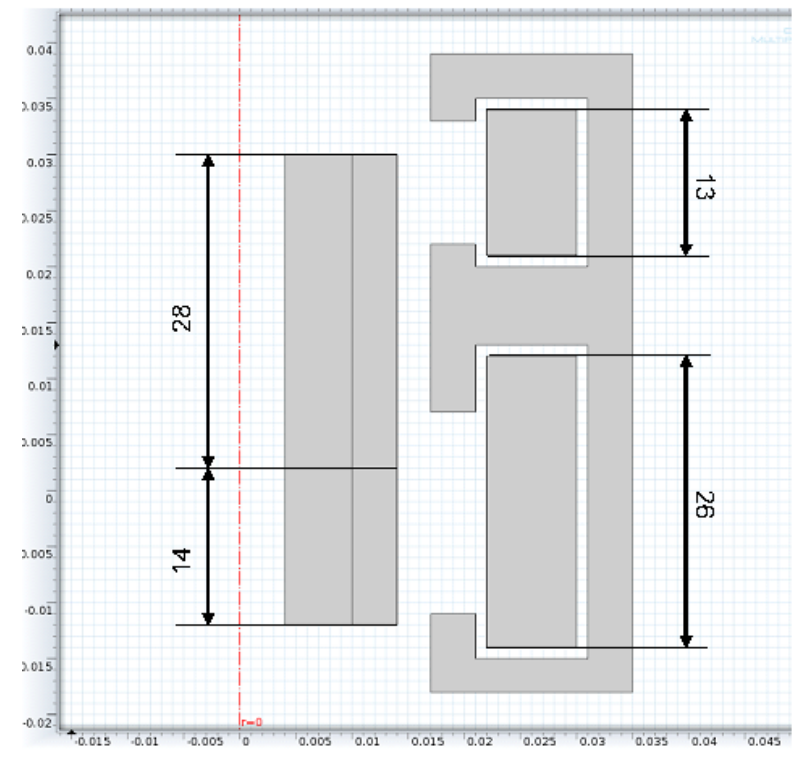

Fig. 12. Geometry of the optimized actuator in the trace of the simulation results.

As can be seen the actuator shown in Fig. 12 has a special geometry, namely: the top coil has a half height from the bottom coil, and the lower magnet is half the height of the upper coil. In addition to this geometry, the coils are wrapped in the opposite direction, and magnets magnetized in opposite directions [5].

\section{Experimental validation of optimization of magnetic circuit geometry}

For the experimental validation of the electromagnetic simulation results and optimization of the magnetic circuit geometry, we have developed an experimental stand for measuring the force according to the air gap and the excitation current. [2]

The stand has 3 main components: support, pedal, linear actuator and other secondary components: limiter, actuator holder and various clamping or limiting parts. The stand is made of aluminum.

The stand is designed to experimentally determine the variation of the electromagnetic force in the pedal according to the values of the actuator supply current. It has a variable power 
supply to the actuator. Depending on the values of the power supply, we have a pedal force that prevents it from being pressed.

The actuator will give a feedback force and will block the pedal movement, the pedal will not be able to feel when it is touched. With the increase or decrease of the current, the electromagnetic force in the pedal will increase or decrease.

The measurements were made using a force cell equipped with position sensors. The program in which the test results were obtained is "Diadem National Instruments" and it displays the force according to the angle of rotation of the actuator sensor in front of the pedal spindle movement. [5]
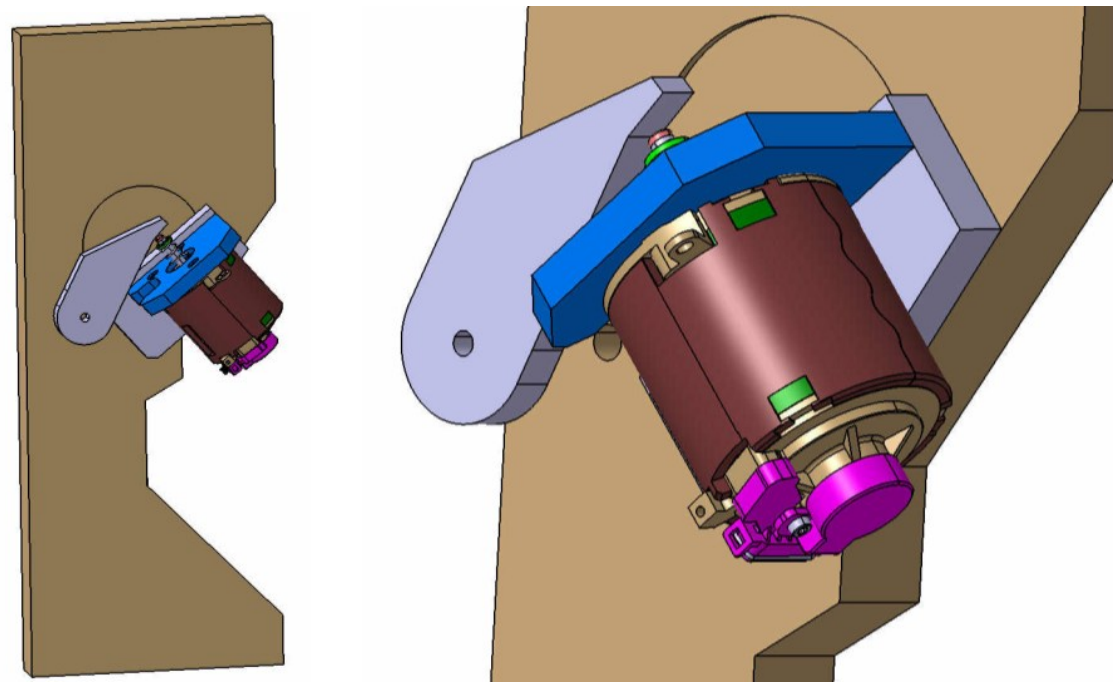

Fig. 13. The Catia V5 project of the force measuring stand.
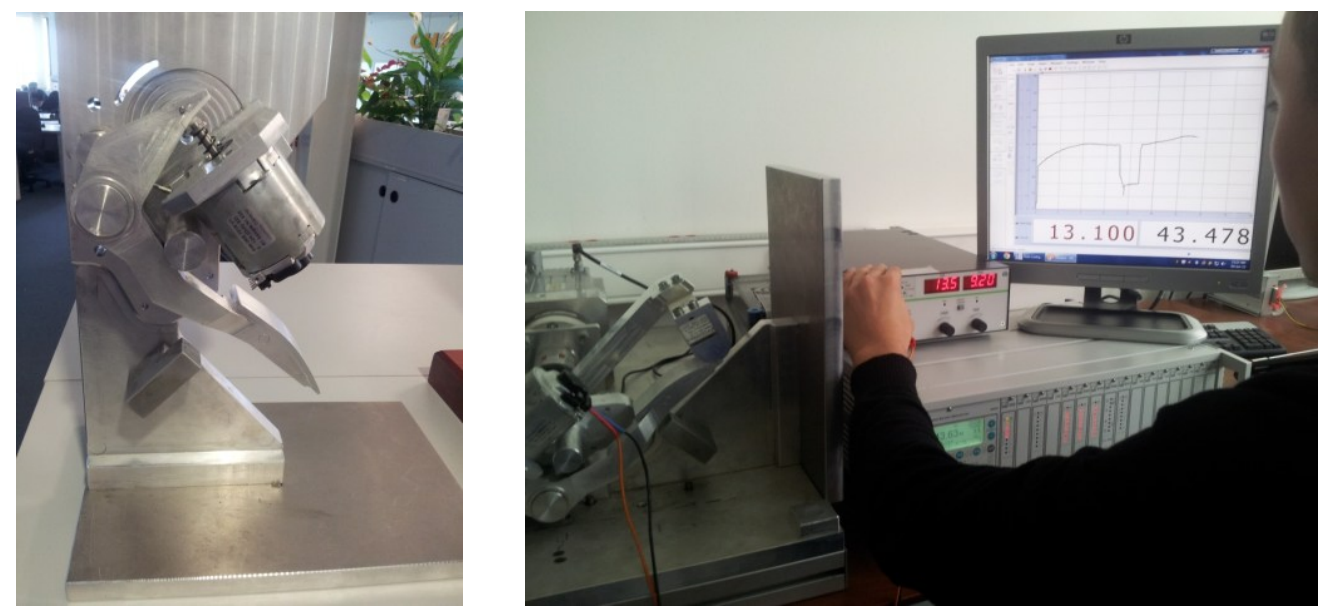

Fig. 14. Experimental force measuring stand.

In figure 16, we represented the variation of force on the 8-12 $\mathrm{mm}$ range, doing so: when the actuator stroke reached $8 \mathrm{~mm}$, we powered from the source with $8 \mathrm{~A}$ on a travel of $4 \mathrm{~mm}$ to $12 \mathrm{~mm}$, after which we stopped supplying it continuing - and thus the free race. The graphs obtained from the tests show that we have closed curves. The explanation is: The upper curve represents the force of the actuator pin movement over a distance of 0-16 $\mathrm{mm}$, and the lower curve that closes the loop is the force developed by returning the pin to the initial position. 
Because of the limitations imposed by the experimental stand, the determination of force was made only for $0 \mathrm{~A}$ (only permanent magnetization), $4 \mathrm{~A}$ and $8 \mathrm{~A}$.

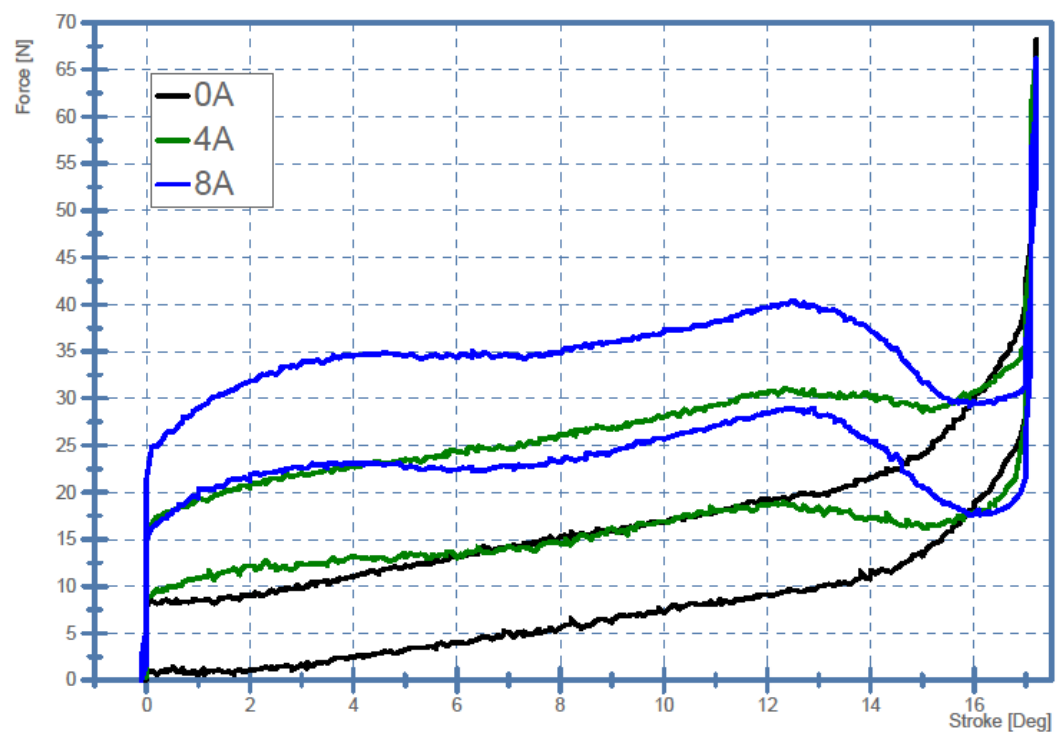

Fig. 15. Force-displacement graph for 0,4 and 8 A obtained from the tests.

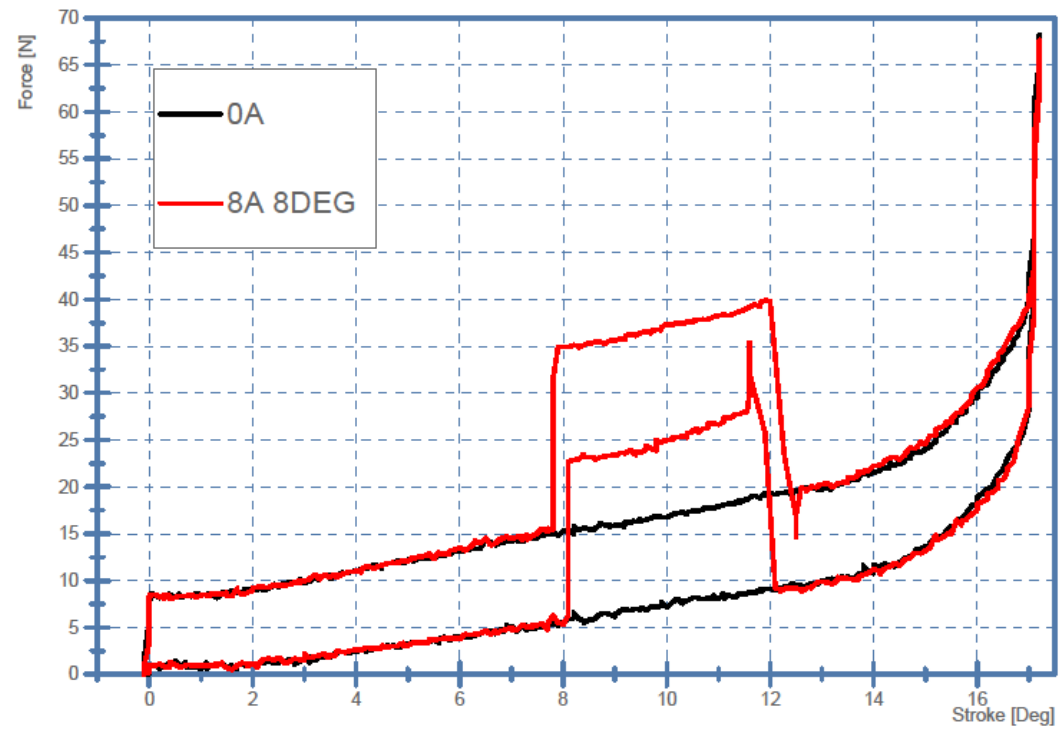

Fig. 16. Driving force-displacement graph with a force of 8-12 mm.

\section{Conclusions}

The method of constructive optimization of an actuator presented in the paper proved to be useful, precise and rapid. We have designed and magnetically simulated an actuator model of a non-existent construction on the current market.

When optimizing the magnetic circuit of the actuator we studied the variation of coil and magnet dimensions, more precisely we performed an actuator model with a $13 \mathrm{~mm}$ coil top coil wound in one direction and a lower double coil $(26 \mathrm{~mm})$ coil wound in opposite way. 
Also, the magnets were constructed of different heights, namely the upper magnet having a height of $28 \mathrm{~mm}$ and radiating magnetically in one direction, the lower one having a height of half a $14 \mathrm{~mm}$ and radiating magnetically in the opposite direction.

Following the magnetic simulation of this actuator model, we have achieved powerdisplacement graphs with line variations for 4, 8, 12 A and the maximum force developed at 12 A being approximately $160 \mathrm{~N}$.

Performing tests for measuring the electromagnetic force on the actual stand for different currents has materialized in force-displacement graphs (in the "Diadem National Instruments" software) which experimentally confirm the results from the simulations and thus the validity of the electromagnetic optimization method.

The electromagnetic simulation must be completed with a thermal simulation to complete the assisted design of the actuator.

\section{Reference}

1. E.G. Thompson Introduction to the Finite Element Method: Theory, Programming and Applications, J. New York, Wiley \& Sons Publishers (2004)

2. L. Popescu, M. Popa, The electromagnetic drive movement-dependent inductivity, International Conference The contribution of Information Tehnology to Science, Economy, Society and Education, T.E.I. of Piraeus", Athens (2007)

3. L. Popescu, Echipamente Electrice, Ed. Alma Mater, Sibiu (2008)

4. G. Dhatt, E. Lefrancois and G. Touzot, Finite element method, John Wiley \& Sons (2012)

5. R. Tahmasebi, H. V. Alizadeh, S. Rahimi, and B. Boulet, Robust ho force control of a solenoid actuator using experimental data and finite element method, Control Applications (CCA) IEEE Conference (2014) 